\title{
Diffusion to infinity for periodic orbits in meromorphic dynamics
}

\author{
by \\ Janina Kotus (Warszawa) and \\ Grzegorz Świątek (University Park, PA)
}

\begin{abstract}
A small perturbation of a rational function causes only a small perturbation of its periodic orbits. We show that the situation is different for transcendental maps. Namely, orbits may escape to infinity under small perturbations of parameters. We show examples where this "diffusion to infinity" occurs and prove certain conditions under which it does not.
\end{abstract}

1. Introduction. The Fatou set $F(f)$ of a meromorphic function $f$ : $\mathbb{C} \rightarrow \widehat{\mathbb{C}}$ is defined in exactly the same manner as for rational functions; $F(f)$ is the set of points $z \in \mathbb{C}$ such that all the iterates are defined and form a normal family on a neighborhood of $z$. The Julia set $J(f)$ is the complement of $F(f)$ in $\widehat{\mathbb{C}}$. Thus, $F(f)$ is open, $J(f)$ is closed, $F(f)$ is completely invariant while $f^{-1}(J(f))=J(f) \backslash\{\infty\}$ and $f(J(f) \backslash\{\infty\}) \subset J(f)$. For description of the dynamics of meromorphic functions see e.g. [5]. We would however like to note that it easily follows from Montel's criterion of normality that if $f: \mathbb{C} \rightarrow \widehat{\mathbb{C}}$ has at least one pole which is not an omitted value then (see [5])

$$
J(f)=\overline{\bigcup_{n \geq 0} f^{-n}(\infty)} .
$$

We define the prepoles of order $p \in \mathbb{N} \cup\{0\}$ as the set

$$
\mathcal{P}_{p}=\left\{z \in \mathbb{C}: f^{p}(z)=\infty\right\}, \quad p \geq 1, \quad \mathcal{P}_{0}=\{\infty\} .
$$

For all meromorphic functions it is known that the Julia set is the closure of the repelling periodic points (see [2], [6], [3]). We want to investigate the structure of the repelling periodic points in the Julia set $J(f)$.

2000 Mathematics Subject Classification: Primary 37F10, 37F45; Secondary 30D05, $32 \mathrm{H} 50$.

Research of J. Kotus was partially supported by the Foundation for Polish Science, Polish KBN Grant No 2 P03A 00917 and TUW Grant no 503G 112000442200.

This work was done in part during G. Świątek's stay at the Mathematical Institute of PAN in Warsaw. His research was also partially supported by an NSF grant DMS-0072312. 
In 1967 Baker conjectured that if $p \geq 2$ then every entire function $f$ has infinitely many periodic points of primitive period $p$. Earlier, he had proved in [1] that there exists at most one positive integer $p$ (depending on $f$ ) for which this fails to be true. Baker's conjecture was proved by Bergweiler [4]. He actually shows that $f$ has infinitely many repelling periodic points with this property. Thus for an entire function the unique accumulation point of repelling periodic cycles of period $p$ is the essential singularity of $f$, i.e. $\infty$. For meromorphic functions with at least one pole which is not an omitted value many prepoles of order $p-1$ are accumulation points of repelling periodic points of period $p$. For more precise description see Lemma 6 in $[5]$.

Diffusion to infinity. For families of rational functions, periodic orbits of any period cannot suddenly appear or disappear. Let $F_{\lambda}$ be a rational function depending analytically on some parameter $\lambda$. If $F_{\lambda_{0}}$ has a periodic point $p_{0}$, then under a small perturbation of $\lambda$ there will be related periodic points nearby; all periodic orbits of a given period for small changes of the parameter are realized in this way. For entire functions, however, it is possible that as the parameter tends to some value $\lambda_{0}$ inside the parameter space, the orbit nevertheless migrates toward $\infty$ so that at $\lambda_{0}$ it has no counterpart. An example is the family $F_{\lambda}(z)=z\left(e^{z}+1-\lambda\right)$ for which $\log \lambda$ is a fixed point which tends to $\infty$ as $\lambda \rightarrow 0$ (this example was suggested by the referee). This appears more likely still for families of meromorphic functions, since all that is needed for a point of an orbit to escape to $\infty$ is that the preceding point should go to a pole.

Since both the repelling periodic orbits and the prepoles are dense in the Julia set, and at first sight one is inclined to think that they move independently with parameter, we should observe many collisions, perhaps on a dense set of parameters. Such collisions are dynamically serious events, since they imply a periodic orbit escaping to $\infty$ and certainly contradict structural stability.

The results of this paper go in two directions. First, we show that under certain conditions which are naturally satisfied by most popular families, diffusion of periodic orbits to $\infty$ may only occur in one case: if a finite asymptotic value of the map hits the pole. This immediately "explains" how structural stability is possible if the asymptotic values are under control. Secondly, we prove that if a post-asymptotic pole is present, the diffusion can naturally occur. This has previously been noticed for attracting orbits (see [7]), and we give an example of disappearing repelling orbits, which is perhaps a more disturbing phenomenon since it happens on the Julia set.

Acknowledgements. We are grateful to the referee for numerous insightful comments. 


\subsection{Setting of the problem}

Hypotheses. Let $\Omega$ be an open subset of $\mathbb{C}$. We consider a family $F: \mathbb{C} \times \Omega \rightarrow \widehat{\mathbb{C}}$ which is continuous, for every fixed $\lambda_{0} \in \Omega$ the map $F\left(\cdot, \lambda_{0}\right)$ is meromorphic in $\mathbb{C}$, and for every fixed $z_{0} \in \mathbb{C}$ the mapping $F\left(z_{0}, \cdot\right): \Omega \rightarrow \widehat{\mathbb{C}}$ is meromorphic (perhaps constantly equal to $\infty$ ).

Uniform pole hypothesis. Given the family $F$ which satisfies the "general hypotheses" specified before, assume additionally that there is a constant $Q$ so that for every compact $K \subset \Omega$ and $N_{0}>0$ there is $M_{0}<\infty$ so that whenever for some $z \in \mathbb{C}$ and $\lambda \in K$,

$$
M_{0}<|F(z, \lambda)|<\infty
$$

then $\left|F_{z}(z, \lambda)\right|>N_{0}$ and $\left|F_{\lambda}(z, \lambda)\right|<Q\left|F_{z}(z, \lambda)\right|$.

These hypotheses were modeled on the families $z \mapsto \lambda \exp (z), \lambda \tan z$, $\lambda \sec z, \lambda \neq 0$, in which cases they are satisfied. For $\lambda \exp (z)$ they hold vacuously, while in the remaining cases the only way for $F(z, \lambda)$ to get near $\infty$ is to pass through a pole, and all these poles satisfy uniform estimates.

Some notations. For $\lambda_{0} \in \Omega$ define a set $\mathcal{A}\left(\lambda_{0}\right) \in \mathbb{C}$ by the condition $a \in \mathcal{A}\left(\lambda_{0}\right)$ if and only if there are continuous functions $X:(0,1) \rightarrow \mathbb{C}$ and $\Lambda:(0,1) \rightarrow \Omega$ so that $\lim _{t \rightarrow 0} X(t)=\infty, \lim _{t \rightarrow 0} \Lambda(t)=\lambda_{0}$ and $\lim _{t \rightarrow 0} F(X(t), \Lambda(t))=a$.

Finite asymptotic values of $F\left(\cdot, \lambda_{0}\right)$ are members of $\mathcal{A}\left(\lambda_{0}\right)$. In simple cases this may be it, on the other hand for $F(z, \lambda)=\lambda z$ the set $\mathcal{A}(0)$ is easily seen to be the whole complex plane.

For a function of several variables, we will consider its iterates with respect to the first variable, regarding the remaining ones as fixed. For example, we will write

$$
F^{3}(x, \lambda):=F(F(F(x, \lambda), \lambda), \lambda) .
$$

Periodic orbits and regularity. A periodic point with period $p \geq 1$ for us will be a pair $(x, \lambda)$ which satisfies the condition $F^{p}(x, \lambda)=x$, whether or not $p$ is minimal with this property.

Definition 1. A parameter $\lambda_{0} \in \Omega$ is considered regular for period $p$ if for every pair of continuous functions $X:(0,1) \rightarrow \mathbb{C}$ and $\Lambda:[0,1) \rightarrow \Omega$ chosen so that for every $t \in(0,1)$ the pair $(X(t), \Lambda(t))$ is a periodic orbit of period $p$ and $\Lambda(0)=\lambda_{0}$, the $\operatorname{limit}_{\lim _{t \rightarrow 0}} X(t):=x_{0} \in \mathbb{C}$ exists and $\left(x_{0}, \lambda_{0}\right)$ is a periodic orbit of period $p$.

Observe that if all values from an open connected set $U \subset \Omega$ are regular for some period $p$, then for every $\lambda_{0}, \lambda_{1} \in U$ and a periodic orbit $\left(x_{1}, \lambda_{1}\right)$ of period $p$, there exist $x_{0} \in \mathbb{C}$ and continuous functions $X:[0,1] \rightarrow \mathbb{C}$, $\Lambda:[0,1] \rightarrow \Omega$ with $X(i)=x_{i}, \Lambda(i)=\lambda_{i}$ for $i=0,1$ and such that for each $t \in[0,1]$ the pair $(X(t), \Lambda(t))$ is a periodic orbit of period $p$. 
Thus, a $\lambda_{0}$ is regular if no periodic orbit disappears as parameter values approach $\lambda_{0}$. On the other hand, if all values in a neighborhood are regular, then every periodic orbit with $\lambda$ in that neighborhood is obtained by perturbing some periodic orbit for $\lambda_{0}$.

Theorem 1. For some $\lambda_{0} \in \Omega$ and $p \geq 1$ suppose that $\mathcal{A}\left(\lambda_{0}\right)$ does not contain prepoles of $F\left(\cdot, \lambda_{0}\right)$ of order less than $p$. Then $\lambda_{0}$ is regular for period $p$.

Examples of the disappearance of periodic orbits

Theorem 2. For the family $F(z, \lambda)=\lambda \tan z, \lambda \neq 0$, which satisfies our hypotheses, for every $p \geq 2$ there exists a parameter value $\lambda_{0}$ such that $F^{p-2}\left(\lambda_{0} i, \lambda_{0}\right)$ is well defined and a pole, and $\lambda_{0}$ is not regular. Moreover, one can pick functions $\Lambda$ and $X$ as in Definition 1 in such a way that $\lim _{t \rightarrow 0} X(t)=\infty$ and either $(X(t), \Lambda(t))$ is an attracting periodic orbit for every $t \in(0,1)$, or it is repelling for every $t$.

2. Proof of regularity. Let us start with a lemma offering a stringent limitation on how an orbit could escape to $\infty$.

Lemma 1. For every period $p \geq 1$ and $\lambda_{0} \in \Omega$ there are an open set $\lambda_{0} \in$ $V \subset \Omega$ and $M<\infty$ and a constant $L$ such that the following property holds: if $\lambda \in V,(x, \lambda)$ is a periodic orbit of period $p$, and $M \leq\left|F^{j}(x, \lambda)\right|<\infty$ for $j=0, \ldots, p-1$, then $\left|\left(F^{p}\right)_{z}(x, \lambda)\right|>2$ and $\left|\left(F^{p}\right)_{\lambda}(x, \lambda)\right|<L\left|\left(F^{p}\right)_{z}(x, \lambda)\right|$.

Proof. We will choose $V$ so that $\bar{V}$ is compact in $\Omega$ and then based on the uniform pole hypothesis we made about $F$, specify $N_{0}=2$ and set $M$ equal to $M_{0}$ postulated by that hypothesis. Now clearly the first claim follows.

The ratio of derivatives $\left(F^{p}\right)_{\lambda}(x, \lambda) /\left(F^{p}\right)_{z}(x, \lambda)$ can be written as

$$
\frac{\left(F^{p}\right)_{\lambda}(x, \lambda)}{\left(F^{p}\right)_{z}(x, \lambda)}=\sum_{j=0}^{p-1} \frac{1}{\left(F^{j}\right)_{z}(x, \lambda)} \cdot \frac{F_{\lambda}\left(F^{j}(x, \lambda), \lambda\right)}{F_{z}\left(F^{j}(x, \lambda), \lambda\right)} .
$$

The first term is bounded by 1 and the ratio of derivatives is bounded by $Q$. The second claim of the lemma follows as well.

Lemma 2. Let $\Lambda:(0,1) \rightarrow \Omega$ be continuous with $\lambda_{0}:=\lim _{t \rightarrow 0} \Lambda(t)$ well defined in $\Omega$. Suppose that there is a continuous function $X:(0,1) \rightarrow \mathbb{C}$ so that for every $t \in(0,1)$ the pair $(X(t), \Lambda(t))$ is a periodic orbit of some fixed period $p$. Then $\lim _{t \rightarrow 0} X(t)$ exists in the Riemann sphere.

Proof. To the contrary, suppose that $X(t)$ has no limit as $t \rightarrow 0$. Consider the set of limit points on the sphere: $z \in L$ if and only if there is a sequence $t_{n} \rightarrow 0$ with $X\left(t_{n}\right) \rightarrow z$. Then $L$ is a non-empty connected set of at least two points, thus of uncountably many. So, there is $z_{0} \in L$ with the property 
that $z_{0}, F\left(z_{0}, \lambda_{0}\right), \ldots, F^{p-1}\left(z_{0}, \lambda_{0}\right)$ are all well defined in $\mathbb{C}$. Then $\left(z_{0}, \lambda_{0}\right)$ has an open neighborhood $U$ in $\mathbb{C} \times \Omega$ such that for every $(z, \lambda) \in U$, $z, \ldots, F^{p-1}(z, \lambda)$ are well defined in $\mathbb{C}$. For a sequence $t_{n} \rightarrow 0$ the points $\left(X\left(t_{n}\right), \Lambda\left(t_{n}\right)\right)$ are in $U$ for almost all $n$.

But then by extracting a subsequence we ensure that $X\left(t_{n_{j}}\right) \rightarrow x_{0}$ and then $\left(x_{0}, \lambda_{0}\right)$ is a periodic orbit of period $p$. But periodic points of period $p$ are isolated in $\mathbb{C}$ for fixed $\lambda_{0}$, so it follows that actually $x_{0}=\lim _{t \rightarrow 0} X(t)$.

Proof of Theorem 1. To check that $\lambda_{0}$ is regular, consider functions $\Lambda$ and $X$ as in Definition 1. By Lemma 2, the functions

$$
X(t), F(X(t), \Lambda(t)), \ldots, F^{p-1}(X(t), \Lambda(t))
$$

all have limits in $\widehat{\mathbb{C}}$. If all these limits are finite, then they form a periodic orbit of $F\left(\cdot, \lambda_{0}\right)$ and so the regularity condition is satisfied.

The case when all these limits are infinite is impossible in view of Lemma 1 . Indeed, then for $t$ small enough all points $X(t), \ldots, F^{p-1}(X(t), \Lambda(t))$ are outside a fixed neighborhood of $D(0, M)$. Then Lemma 1 implies that $X(t)$ depends holomorphically with bounded derivative on $\Lambda(t)$, which means that it cannot escape to $\infty$.

Hence, some points of the orbit tend to $\infty$, while others have finite limits. Without loss of generality, let $\lim _{t \rightarrow 0} X(t)=a$ be finite, while $\lim _{t \rightarrow 0} F^{p-1}(X(t), \Lambda(t))=\infty$. Then $a \in \mathcal{A}\left(\lambda_{0}\right)$, and its $(p-1)$ st image under $F\left(\cdot, \lambda_{0}\right)$ is not defined in the plane (perhaps an earlier image is already undefined). So, the hypothesis of Theorem 1 is violated.

3. Examples. We will now prove Theorem 2. In the case when $(X(t), \Lambda(t))$ are attracting, an example was exhibited in [7]. So we will concentrate on constructing a repelling example. The example will be found in the family $F(z, \lambda)=\lambda \tan z$ where $\lambda \in \Omega:=\mathbb{C} \backslash\{0\}$. We choose $\lambda_{0}$ so that the asymptotic value $\lambda_{0} i$ gets to a pole of $\tan z$ after exactly $p-2$ steps, with some $p \geq 2$.

When $\lambda$ is close to $\lambda_{0}$, we can find a unique $v(\lambda)$ close to 0 with the property that $F^{p-2}(\lambda(i+v(\lambda)))$ is a pole of $\tan z$. The dependence $\lambda \mapsto v(\lambda)$ is holomorphic, but it could have a critical point at $\lambda_{0}$. With this caveat, we can use $v$ to locally reparametrize a neighborhood of $\lambda_{0}$.

For an integer $n$ let $S_{n}$ denote the set of $z \in \mathbb{C}$ with $\Im z>1$ and $\Re z \geq \pi n$.

Lemma 3. There exist a fixed $\varepsilon_{0}>0$ and a neighborhood $W$ of $\lambda_{0}$ such that for every $\varepsilon \in\left(0, \varepsilon_{0}\right)$ there exists $N$ so that the following conditions hold provided that $\lambda \in W$ and $\varepsilon_{0} \geq|v(\lambda)|>\varepsilon$ :

- for every $n \geq N$ there exists a point $s \in S_{n}$, periodic with period $p$ under $F(\cdot, \lambda)$ and such that $\tan s \in D(i+v(\lambda),|v(\lambda)| / 2)$, 
- if $s \in S_{N}$ is periodic with period $p$ under $F(\cdot, \lambda)$ and

$$
\tan s \in D(i+v(\lambda),|v(\lambda)| / 2),
$$

then $\left|\left(F^{p}\right)_{z}(s, \lambda)\right| \geq 2$ and $\tan s \in D(i+v(\lambda),|v(\lambda)| / 4)$.

Proof. For $\lambda$ near $\lambda_{0}, F^{p-1}(\cdot, \lambda)$ maps $D(i+v(\lambda),|v(\lambda)| / 2)$ into the complement of $D\left(0, L_{1}|v(\lambda)|^{-1}\right)$, and $D(i+v(\lambda),|v(\lambda)| / 4)$ onto the complement of $D\left(0, L_{2}|v(\lambda)|^{-1}\right)$, where $L_{1}, L_{2}$ are positive constants. On the other hand, $S_{n} \backslash S_{n+1}$ maps onto a fixed neighborhood of $i$ under $\tan z$ for every $n$. It follows that the choice of $N>L_{1} \varepsilon^{-1}$ implies that $F^{p}(\cdot, \lambda)$ maps $D(i+v(\lambda),|v(\lambda)| / 2)$ onto a set containing the closure of this disk in a one-toone fashion, hence implying the existence claim. On the other hand, choosing $N>L_{2} / \varepsilon$ implies that $\tan p$ is actually in $D(i+v(\lambda),|v(\lambda)| / 4)$ and, since this disk is mapped by $F^{p}(\cdot, \lambda)$ onto a set containing $D(i+v(\lambda),|v(\lambda)| / 2)$, this implies the expansion claim as well.

Now let us specify some small $\varepsilon$, adjust $N$, and pick initial $\lambda_{1}$ for which $\left|v\left(\lambda_{1}\right)\right|>\varepsilon$. Suppose that $s \in S_{n}, n \geq N+5$; such an initial point can always be found from the existence part of Lemma 3 . As we vary $\lambda$, we get, at least locally, a holomorphic arc of repelling periodic points $s(\lambda)$. By Lemma 3, as long as $s(\lambda) \in S_{N}$, we have $\tan s(\lambda) \in D(i+v(\lambda),|v(\lambda)| / 4)$ and $\left|\left(F^{p}\right)_{z}^{\prime}(s(\lambda), \lambda)\right| \geq 2$.

First, perturb $\lambda$ in such a way that $|v(\lambda)|$ remains fixed but $\arg v(\lambda)$ changes by $4 \pi$. Let $\lambda(t)$ for $0 \leq t \leq 1, \lambda(0)=\lambda_{1}$, be the corresponding path in the parameter space. Since $\arg v(\lambda)$ changes by $4 \pi, \arg (\tan (s(\lambda(t)))-i)$ has changed by no more than $\frac{9}{2} \pi$ at any point $t_{0}$ during the perturbation. Thus, $s\left(\lambda\left(t_{0}\right)\right)$ is in $S_{N}$, so that the whole perturbation is well defined.

Moreover, as $t$ varies from 0 to $1, \arg (\tan (s(\lambda(t)))-i)$ varies by at least $\frac{7}{2} \pi$ and so $m \neq n$ after the complete perturbation. Choosing the direction of rotating $v(\lambda)$ appropriately, we can ensure that $s(\lambda(1)) \in S_{n+1}$.

Another perturbation consists in moving $v(\lambda)$ radially toward 0 . An argument similar to the previous construction shows that as long as $|v(\lambda)|>\varepsilon$ we have $s(\lambda(t)) \in S_{n-1}$ provided that $s(\lambda(0)) \in S_{n}$.

Combining these two perturbations we can crash $v(\lambda)$ into 0 all the time moving through orbits of the type $s(\lambda)$. Indeed, we shrink $v(\lambda)$ radially as long as possible using the second perturbation. Using the first perturbation we can then increase $n$ as much as needed without changing $|v(\lambda)|$ until it becomes more than $N(\varepsilon / 100)+6$. This allows us to again shrink $|v(\lambda)|$ a hundred times while varying $n$ by no more than 1 . Combining the perturbations in this way we allow $\lambda$ tend to $\lambda_{0}$. Since $\tan s(\lambda)$ converges to $i, s(\lambda)$ itself goes to $\infty$ and thus the proof of Theorem 2 is complete. 


\section{References}

[1] I. N. Baker, The existence of fixpoints of entire functions, Math. Z. 73 (1960), 280-284.

[2] —, Repulsive fixpoints of entire functions, ibid. 104 (1968), 252-256.

[3] I. N. Baker, J. Kotus and Y. Lü, Iterates of meromorphic functions: I, Ergodic Theory Dynam. Systems 11 (1991), 241-248.

[4] W. Bergweiler, Periodic points of entire functions: proof of a conjecture of Baker, Complex Variables 12 (1991), 57-72.

[5] - Iteration of meromorphic functions, Bull. Amer. Math. Soc. 29 (1993), 151-188.

[6] P. Bhattacharyya, Iteration of analytic functions, $\mathrm{PhD}$ thesis, Univ. of London, 1969.

[7] L. Keen and J. Kotus, Dynamics of the family $\lambda \tan (z)$, Conformal Geometry Dynam. 1 (1997), 28-57.

Department of Mathematics

Warsaw University of Technology

00-661 Warszawa, Poland

E-mail: janinak@impan.gov.pl
Department of Mathematics Penn State University University Park, PA 16802, U.S.A.

E-mail: swiatek@math.psu.edu

Received 27 November 2001;

in revised form 28 February 2002 